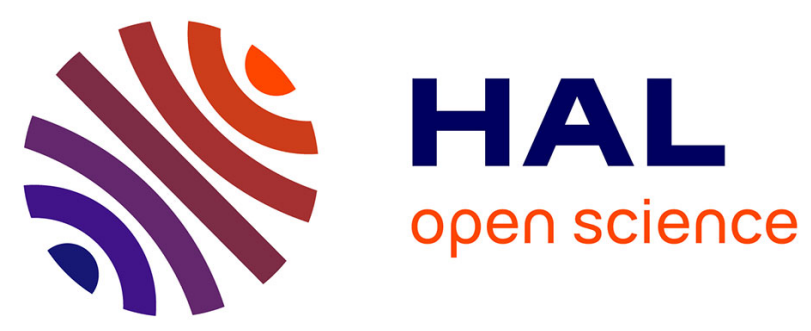

\title{
Controlling a multi-joint arm actuated by pneumatic muscles with quasi-DDP optimal control
}

\author{
Ganesh Kumar Hari Shankar Lal Das, Bertrand Tondu, Florent Forget, \\ Jérôme Manhes, Olivier Stasse, Philippe Souères
}

\section{To cite this version:}

Ganesh Kumar Hari Shankar Lal Das, Bertrand Tondu, Florent Forget, Jérôme Manhes, Olivier Stasse, et al.. Controlling a multi-joint arm actuated by pneumatic muscles with quasi-DDP optimal control. IEEE/RSJ International Conference on Intelligent Robots and Systems (IROS 2016), Oct 2016, Daejon, South Korea. 10.1109/IROS.2016.7759103 . hal-01432100

\section{HAL Id: hal-01432100 https://hal.science/hal-01432100}

Submitted on 11 Jan 2017

HAL is a multi-disciplinary open access archive for the deposit and dissemination of scientific research documents, whether they are published or not. The documents may come from teaching and research institutions in France or abroad, or from public or private research centers.
L'archive ouverte pluridisciplinaire HAL, est destinée au dépôt et à la diffusion de documents scientifiques de niveau recherche, publiés ou non, émanant des établissements d'enseignement et de recherche français ou étrangers, des laboratoires publics ou privés. 


\title{
Controlling a multi-joint arm actuated by pneumatic muscles with quasi-DDP optimal control
}

\author{
G. Kumar Hari Shankar Lal Das ${ }^{1,2}$, B. Tondu ${ }^{1,2}$, F. Forget ${ }^{1}$, J. Manhes ${ }^{1}$, O. Stasse ${ }^{1}$, P. Soueres ${ }^{1}$
}

\begin{abstract}
Pneumatic actuators have inherent compliance and hence they are very interesting for applications involving interaction with environment or human. But controlling such kind of actuators is not trivial. The paper presents an implementation of iterative Linear Quadratic regulator (iLQR) based optimal control framework to control an anthropomorphic arm with each joint actuated by an agonist-antagonistic pair of Mckibben artificial muscles. The method is applied to positioning tasks and generation of explosive movements by maximizing the link speed. It is then compared to traditional control strategies to justify that optimal control is effective in controlling the position in highly non-linear pneumatic systems. Also the importance of varying compliance is highlighted by repeating the tasks at different compliance level. The algorithm validation is reported here by several simulations and hardware experiments in which the shoulder and elbow flexion are controlled simultaneously.
\end{abstract}

\section{INTRODUCTION}

Pneumatic actuators are inherently compliant and have very high power to weight ratio. These factors motivate researchers to use pneumatic actuation for exoskeleton, prosthesis, rehabilitation or even in walking robot and human robot interaction. The goal of this paper is to use an arm actuated by McKibben muscles to perform explosive tasks like hammering or kicking balls. Recent works [1] advocates other kind of actuation system which are easier to control but come with a high price tag. Our goal was to demonstrate here that with minimal modification of an existing platform [2], it is possible to perform such kind of explosive motions, and not fine manipulation as in [1]. The Mckibben artificial muscle is known for its nonlinearities and hence pose a great control challenge. These non-linearities are mainly due to hysteresis, saturation and internal friction between fabrics. So far these challenges were dealt with traditional controllers like high gain PID controller, sliding mode controller for position control [3]. These methods usually lead to stiff system dynamics with higher impedances. Recent developments in pneumatic actuation control [4], [5] are use Model Predictive Control (MPC). For instance in [6], the control scheme is based on a switching Piece Wise Affine (PWA) system model approximation. The method is able to capture the high non-linearities of the Pneumatic Artificial Muscle (PAM). In [4] a linear formulation of the actuation system is proposed to simplify the algorithm implementation and makes it real-time.

The contribution of this paper is two fold.

- First it proposes a new model which provides a good compromise between accuracy and simplicity.

\footnotetext{
${ }^{1}$ are with

CNRS, LAAS, 7 avenue du colonel Roche, F-31400 Toulouse, France

2 are also with Univ de Toulouse, INSA, F-31400 Toulouse, France

gkharish, btondu, ostasse, psoueres@laas.fr
}

- The second contribution is to show that, on this model an iLQR control scheme can be used to generate with good precision positioning task and explosive movements.

This is achieved with the introduction of an empirical model of pressure generation from the Intensity-Pressure converter $(\mathrm{I} / \mathrm{P})$. Each of the seven Degrees of Freedom (DoFs) of the manipulator arm of LAAS-CNRS is actuated by a pair of agonist-antagonist Mckibben muscles. The capabilities of the non-linear iLQR control to execute some simple tasks of reaching a point, maximizing the velocity of joint and end effector are demonstrated on this platform.

The paper is organised as follows: The dynamical model of joints actuated by Mckibben muscles is presented in section II. Section III describes the model of the anthropomorphic robot arm. The optimal control formulation and brief introduction to iterative Linear Quadratic Regulator(iLQR) is presented in section IV. Section V presents simulations and experiments.

\section{DYNAMIC MODEL OF A JOINT ACTUATED BY MCKIBBEN MUSCLES}

There have been several attempts to model the $\mathrm{McK}$ ibben artificial muscle [4], [7], [8], [9], [10]. The difficulties for getting an accurate model are due to a combination of complex phenomena during static and dynamic contraction: shape changing at the muscle tip which lose their initial cylindrical shape for a conical one, mobility and flexibility of the braided sleeve, elasticity of the inner rubber tube, exotic friction in the textile braided sleeve without forgetting, for the pneumatic version of the McKibben muscle, dynamic fluidic phenomena resulting from the artificial muscle volume variation during contraction. When we want to include such a physical model into the closed-loop control of a McKibben muscle actuator, a compromise must be found between the complexity of an accurate model and the time required for its computation. The need for an efficient but not too complex dynamic muscle model is all the more important in the case of artificial muscles for robot arms. Indeed the preservation of the actuator compliance imposes a joint direct-drive mode and so the consideration of a dynamic robot model, linked to the actuator model. The originality of our approach, described in this section, consists in including an original model of real pressure variation inside a simple McKibben muscle dynamic model before proposing a multi-variable pressure control of the actuator made of two similar antagonist muscles.

\section{A. Muscles side dynamics}

The following model was considered for relating the dynamic contraction force $F$ of the artificial muscle to its control pressure $P$ and contraction ratio.

$$
F(\varepsilon, P)=\left(\pi r_{0}^{2}\right) P\left[a(1-k \varepsilon)^{2}-b\right]
$$




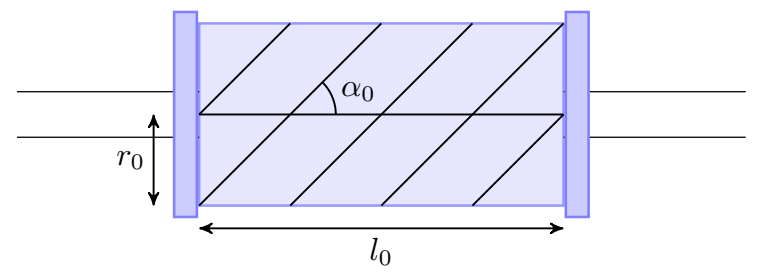

Fig. 1. Muscle geometric parameters: $l_{0}$ initial length, $r_{0}$ radius and $\alpha_{0}$ braid angle

Where,

$$
\begin{array}{r}
\varepsilon=\frac{l_{o}-l}{l_{o}} \\
a=\frac{3}{\tan ^{2} \alpha_{o}} \\
b=\frac{1}{\sin ^{2}\left(\alpha_{o}\right)}
\end{array}
$$

$l$ being the current muscle length and $l_{o}, r_{o}, \alpha_{o}$ are the muscle geometric parameters corresponding to its initial length, radius and braid angle respectively (see [7] for more details). The parameter $k$, slightly greater than 1 , is empirically chosen for taking into account the conic shape at muscle tips. The viscous coefficient is also experimentally estimated for expressing the naturally damped behavior of the artificial muscle contraction due to the complex kinetic friction inside the braided sleeve. Although hysteresis phenomenon due to dry friction is not included in this model, we think it captures most of the static and dynamic behaviour of the artificial muscle.

\section{B. Pressure side dynamics}

Control pressure in a muscle is provided by an IntensityPressure (I/P) converter which translates a current value into a desired pressure value that has to be fed to the muscle. In literature, see [11] for a survey, there exist several models of pressure generated in a muscle in terms of the mass of air injected, volume of the muscle and some parameters of servo valves or I/P converter (for an example see [12]. These models are highly non-linear and eventually become too cumbersome for devising control strategies. We propose a somewhat simpler empirical model to cover the dynamics of the pressure generation from the I/P converter. It is derived from SAMSON I/P converters reported in [13]. Following this model the instantaneous pressure $p$ inside a muscle is represented by a damped second order differential function with a control pressure as input as follows:

$$
\ddot{p}+2 w_{n} \dot{p}+w_{n}^{2} p=w_{n}^{2} p_{\text {des }}
$$

Note that the input of the intensity converter is a current value which is then scaled to the corresponding pressure control input $P_{d e s}$ in the pressure unit. The above equation is also non-linear as the natural frequency $w_{n}$ has been empirically identified as a function of the volume of the muscle, $V$.

$$
w_{n}=2 \pi f_{v}(1 / V)
$$

$f_{v}$ is the empirically found parameter. The volume of a McKibben artificial muscle can be approached by the following cylindrical volume: $V=\pi r^{2} l$, where $r$ and $l$ are the current radius and length of the artificial muscle. By using the relationship Eq.(4) as reported in [14], we deduce an expression for the volume of the muscle.

$$
\begin{gathered}
r / r_{0}=\left(1 / \sin \left(\alpha_{o}\right)\right) \sqrt{\left.(1-\varepsilon)^{2}\right)} \\
V=\frac{\pi r_{o}^{2} l}{\sin ^{2}\left(\alpha_{o}\right)}\left(1-\cos ^{2}\left(\alpha_{0}\right)(1-\varepsilon)^{2}\right)
\end{gathered}
$$

For a second order system, the rise time is inversely proportional to its natural frequency and in the case of our pressure dynamic model, the natural frequency is inversely proportional to the volume. It implies that the bigger the muscle, the larger the rise time. Fig. 2 depicts a typical variation of $w_{n}$ with the joint angle at one of the manipulator joints. If the range
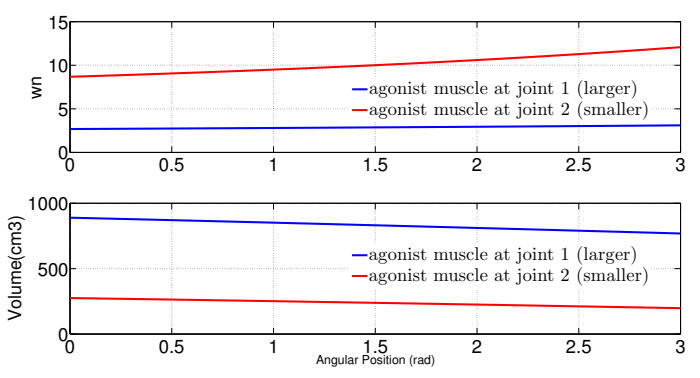

Fig. 2. Natural frequency and volume of the muscle with the joint angle

of operation for a joint is small i.e range of $\theta$ is small, then the corresponding changes in the volume of the muscles as $\theta$ varies, would be small at that joint. In such case, a constant value for $w_{n}$ could be chosen for the muscles corresponding to their mean volumes. Here, another interesting point to notice is that for different muscles, pressure dynamics of a larger volume muscle will be slower. The above two points help in trading off between the operating range of the joint and the corresponding easiness of control strategy.

\section{Agonistic-antagonistic joint actuator}

Following the human arm model, a pair of artificial muscles can be set up in antagonistic fashion to drive a chained wheel of radius R. According to Fig. 3, the resulting actuator torque $\mathrm{T}$ can be written as follows:

$$
T=R\left[F_{1}\left(\varepsilon_{1}, P_{1}\right)-F_{2}\left(\varepsilon_{2}, P_{2}\right)\right]
$$

where $F_{1}$ and $F_{2}$ are the forces of muscles 1 and 2 respectively, defining the antagonist muscle pair. As illustrated in Fig. 3, the relationship between $\theta$, the actuator angle and the contraction ratios for each muscle can be expressed as follows: $\varepsilon_{1}=\varepsilon_{10}+\frac{R \theta}{l_{0}}$ and $\varepsilon_{2}=\varepsilon_{20}-\frac{R \theta}{l_{0}}$, where $\varepsilon_{10}$ and $\varepsilon_{20}$ are the initial contraction ratios for muscle 1 and muscle 2 respectively corresponding to the zero-angular position. Moreover, we propose to specify the pressures in muscle 1 and muscle 2 as follows: $P_{1}=P_{10}+\Delta P_{1}$ and $P_{2}=P_{20}+\Delta P_{2}$ where $P_{10}$ and $P_{20}$ are respectively the initial pressure in the agonist-antagonist muscle and related to zero positioning of the joint. $\Delta P_{1}$ and $\Delta P_{2}$, are the control pressures.

Using Eq.(1), and neglecting the terms $\varepsilon_{1}^{2}$ and $\varepsilon_{2}^{2}$, the torque at the joint can be expressed as

$$
T=K_{1} \Delta P_{1}-K_{2} \Delta P_{2}-K_{3}\left(P_{1}+P_{2}\right) \theta-K_{4} \dot{\theta}+K_{5},
$$




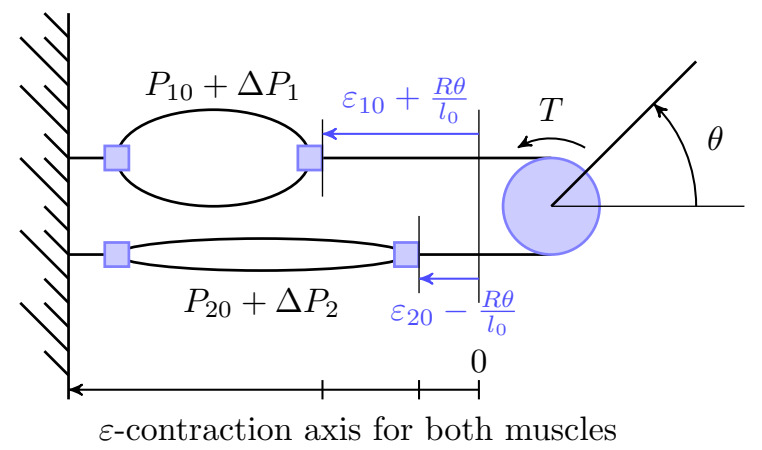

Fig. 3. Pulley-chain driven antagonist muscle actuator made of two identical McKibben artificial muscles. It has the possibility to adapt initial torque at initial zero $\theta$-position by means of $P_{10}, P_{20}, \varepsilon_{10}$ and $\varepsilon_{20}$

where,

$$
\begin{array}{r}
K_{1}=\left(\pi r_{o}^{2}\right) R\left[a\left(1-2 k \varepsilon_{10}\right)-b\right], \\
K_{2}=\left(\pi r_{o}^{2}\right) R\left[a\left(1-2 k \varepsilon_{20}\right)-b\right], \\
K_{3}=2\left(\pi r_{o}^{2}\right) R^{2} k a / l_{o}, \\
K_{4}=\left(\pi r_{o}^{2}\right) R^{2} f_{v} / l_{o}, \\
K_{5}=\left(\pi r_{o}^{2}\right) R\left[(a-b)\left(P_{10}-P_{20}\right)-2 k a\left(P_{10} \varepsilon_{10}-P_{20} \varepsilon_{20}\right)\right] .
\end{array}
$$

If $P_{10}=P_{20}$ and $\varepsilon_{10}=\varepsilon_{20}, K_{5}$ is equal to zero. The details of our robot-arm including the dimensions of muscles are given in [2]. The antagonist muscle actuator is now considered as a MIMO-system whose inputs are the control pressures $\Delta P_{1}, \Delta P_{2}$, and outputs are both the $\theta$ and the actuator stiffness which is defined as the instantaneous ratio between the current torque variation and the current angular position variation (see Eq.(9)). When no gravity effect is considered, the static equilibrium position of the actuator can be directly derived from Eq.(7) with zero angular velocity.

$$
\theta_{\text {equ }}=\left(K_{1} \Delta P_{1}-K_{2} \Delta P_{2}+K_{5}\right) /\left(K_{3}\left(P_{1}+P_{2}\right)\right)
$$

With associated stiffness $\sigma_{\text {equ }}$ expressed as

$$
\sigma_{e q u}=-\frac{\partial T}{\partial \theta}=K_{3}\left(P_{1}+P_{2}\right)
$$

From Eq.(8) and Eq.(9), it is possible to remark that the equilibrium position can be changed while keeping the same stiffness by modulating $\Delta P_{1}$ and $\Delta P_{2}$ with a constant $\Delta P_{1}+\Delta P_{2}$. In the case of a symmetrical pressure variation in both muscles: $\Delta P_{1}=-\Delta P_{2}=\Delta P$, the actuator becomes a SISO-system whose corresponding torque $T_{S I S O}$, is now given by the following relationship:

$$
T=\left(K_{1}+K_{2}\right) \Delta P-K_{3}\left(P_{10}+P_{20}\right) \theta-K_{4} \dot{\theta}+K_{5}
$$

where stiffness at equilibrium position is now constant and equal to $K_{3}\left(P_{10}+P_{20}\right)$. When the joint angle $\theta$ varies, and assuming that the actuator chain is inextensible, the contraction ratio of each muscle is known and, consequently, the pressure dynamic model, proposed in II-B, can be applied to each muscle. It is important to note that, in the case of relatively small joint muscles, the effect of volume variation with joint angle on pressure dynamics could be negligible. If the range of operation is small, a constant $w_{n}$ could be chosen for that joint.
As previously noted, pressure dynamics of a larger volume muscle will be slower which is, especially, the case of the muscles at the shoulder joint compared to the muscles at the elbow joint.

\section{ROBOT DYNAMICS}

This section presents the robot model formally including the rigid body model of the robot with its pressure dynamics. Let us consider a $n$ degrees of freedom robot with generalized joint angle coordinates $q \in \mathbb{R}^{n}$. Each joint is actuated by 2 pneumatic muscles, so there will be $2 n$ muscles, each one with a pressure $P_{i} \in \mathbb{R}^{2 n}$. The robot dynamics can be represented in standard form as below:

$$
\begin{gathered}
M(q) \ddot{q}+C(q, \dot{q})+G(q)=T(q, P) \\
\ddot{P}+2 C_{p} \dot{P}+G_{p} P=G_{p} P_{d e s}
\end{gathered}
$$

$M(q) \in \mathbb{R}^{n \times n}$ is the mass inertia matrix of the robot, $C(q, \dot{q}) \in$ $\mathbb{R}^{n \times 1}$ is the coriolis and centrifugal terms, $G(q) \in \mathbb{R}^{n}$ is the gravity related terms, $T(q, P) \in \mathbb{R}^{n}$ is the torque generated by pneumatic muscles as described in Eq.(6). $C_{p}=$ $\operatorname{diag}\left[w_{n_{1}}, w_{n_{2}}, \ldots w_{n_{2 n}}\right]$ and $G_{p}=\operatorname{diag}\left[w_{n_{1}}^{2}, w_{n_{2}}^{2}, \ldots w_{n_{2 n}}^{2}\right]$ are the collection of coefficients of the pressure dynamics of each muscle and $w_{n_{i}}$ is the natural frequency of the pressure dynamics at the $i^{t h}$ muscle. $P=\left[P_{1}, P_{2} \ldots . . P_{2 n}\right]^{T} \in \mathbb{R}^{2 n}$ is the vector of current muscle pressure and $P_{\text {des }}=\left[P_{\text {des }_{1}}, P_{\text {des }_{2}} \ldots . . P_{\text {des }_{2 n}}\right]^{T} \in$ $\mathbb{R}^{2 n}$, is the vector of desired pressure $P_{\text {des }}$, the control input in pressure unit given to the I/P converter of the $i^{\text {th }}$ muscle. Any reference trajectory in $q$ given to the robot has to respect the pressure dynamics whose bandwidth depends on $w_{n}$. As discussed in the previous section, if the joint is actuated by larger muscles (having smaller bandwidth) and higher rise time, rapidly varying trajectory would be difficult to track and pose a limiting factor in performing explosive tasks. Limits to the operating range of the joints are obviously decided by the maximum contraction of the spanning muscles. In addition to that, $w_{n}$ could be reasonably considered as constant in a limited operating range, removing in this way the non-linearity in pressure dynamics. Apart from this, due to hardware limitation of I/P converter, the control input $P_{d e s}$ is bounded as follows:

$$
U=P_{\text {des }} \in \mathbb{R}^{2 n}: P_{\text {des }} \in\left[P_{\text {min }}, P_{\text {max }}\right]
$$

Where, $P_{\min }, P_{\max }$ are the lower and upper bound on control input $P_{\text {des }}$.

\section{OPTIMAL CONTROL FORMULATION}

This section presents the optimal computational framework used to find the control sequences to perform a desired task. The optimal control problem is the minimization or maximization of a performance criterion with respect to the control under a set of constraints that arise from the physical limitation of the control action and from the plant dynamics.

\section{A. State space representation}

Let us represent the dynamics stated in Eq.(11) and Eq.(12) in state space form considering the state vector as $x=[q, \dot{q}, P, \dot{P}]^{T}$. 


$$
\dot{x}=f(x, u)=\left[\begin{array}{c}
\dot{q} \\
M^{-1}(-C(q, \dot{q})-\dot{-} G(q)+T(q, P)) \\
\dot{P} \\
-C_{p} \dot{P}-G_{p} P+G_{p} u
\end{array}\right]
$$

where, $f$ is the non-linear function given by Eq.(14) in state $x$ and control $u$ that gathers Eq.(11) and Eq.(12). In the present work, we consider the constraints on the state in the optimal control formulation which is discussed in the following subsection.

\section{B. Treatment of Constraints}

There exists a mechanical limit to each degrees of freedom of the arm. To have safe operation, we have introduced these limits as constraints on the state space inside the cost function of the optimal control formulation. The chosen cost function for the state space constraints are expressed in the following equations:

$$
\begin{aligned}
\max & =1-\lambda\left(x_{\max }-x\right) \\
\min & =1-\lambda\left(x-x_{\min }\right) \\
C_{s} & =e^{\lambda * \max }+e^{\lambda * \min }
\end{aligned}
$$

where $x_{\min }, x_{\max }$ are lower and upper limits on the state and $\lambda$ is a constant. The above consideration for the cost function will ensure that the cost near the limits will be very high as evident in the Fig: 4 and hence the optimal solution will keep the system within the operating limits. Also, the control action

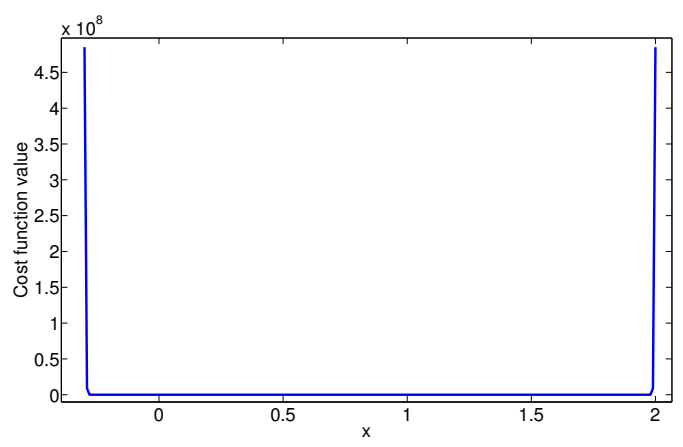

Fig. 4. Cost function of the state constraints

has to be admissible, i.e $u \in U=\left[P_{\min }, P_{\max }\right]$. In order to handle the constraints on the control, the control limited Differential Dynamic Programming (DDP) approach reported in [15] is used. The control problem formulation is then expressed as determining an open-loop control input $u=u(t, x) \in U$ which can minimize or maximize a cost function along a given time interval $t \in[0, T]$ and with initial state $x(0)=x_{0}$. The most generic expression for the cost function can be written as follows:

$$
J\left(x_{0}\right)=C_{f}+C_{r}+C_{s}
$$

where $C_{f}=h(x(T))$ is the final cost, $C_{r}=$ $\int_{0}^{T} c(x(t), u(t, x(t))) d t$ is the integral of the running cost $c(x, u)$ which encapsulates the task objectives and $C_{s}$ is the cost value imposed by the constraints. For a non-linear dynamics Eq.(14) and non-quadratic cost Eq.(16), optimal control solutions can be obtained using full DDP. However, as DDP is computationally expensive, an iterative LQR (iLQR) approach is considered [16]. The $\mathrm{iLQR}$ method is relying on linearizing the dynamics and approximating the cost function to quadratic form along the $x$ trajectory. This control approach is briefly summarized in the next section.

\section{Iterative Linear Quadratic Regulator (iLQR)}

iLQR is initialized with a nominal control sequence and the corresponding state trajectory $\left(x_{0}, u_{0}\right)$. the dynamical system is then linearized as in Eq.(17) and the cost function is approximated by the quadratic form Eq.(18) and a local LQR problem is then solved. Using this solution, the states and the control sequence are improved iteratively.

$$
\begin{gathered}
\dot{\delta} x=A \delta x+B \delta u \\
\Delta J=h_{x}^{T} \delta x(T)+\delta x^{T}(T) h_{x x} \delta x(T) \\
+\int_{0}^{T} c_{x}^{T} \delta x+c_{u}^{T} \delta u+\delta x^{T} c_{x x} \delta x+\delta x^{T} c_{x u} \delta u+\delta u^{T} c_{u u} \delta u
\end{gathered}
$$

where $A=\frac{\partial f}{\partial x}$ and $B=\frac{\partial f}{\partial u}$. In Eq.(17) and Eq.(18) subscript $x$ and $u$ indicate that the function is partially derivated with respect to $x$ and $u$. At every iteration, Eq.(17) and Eq.(18) are solved and $(\delta x, \delta u)$ are deduced from the resolution of a modified Ricatti-type system. Then the new improved sequence is generated by $x \leftarrow x+\delta x$ and $u \leftarrow u+\delta u$. When $\Delta J \approx 0$, the iLQR converges and gives an optimal control sequence $u^{*} \in U$ and the corresponding optimal state trajectory $x^{*}$.

\section{Simulation AND ExPerimental RESUlts}

The experimental set up considered here is the manipulator of LAAS CNRS which is an anthropomorphic arm of seven degrees of freedom $(\mathrm{DoF})$, where each joint is pneumatically actuated by a pair of Mckibben muscles. In the experiment presented in this paper, only two joints are controlled. These joints are the flexion $\theta_{1}$ at the shoulder and the flexion $\theta_{2}$ at the elbow. (see Fig. 5). So, for the experiments, the robot can be viewed as a 2 DoF manipulator with state defined by $x=\left[\theta_{1}, \dot{\theta}_{1}, \theta_{2}, \dot{\theta}_{2}\right]^{T}$. Each muscle is pressurized by an $\mathrm{I} / \mathrm{P}$ converter which converts a current command to a reference pressure value. The objective of the experiments is to evaluate the performance of the iLQR control to achieve the following tasks with our multi-link pneumatic robot.

1) Final position control: We aim to compare the quality of the positioning control in the presence of different loads with the iLQR approach and with the feed-forward proportional control. 2) Capability to execute explosive movements by maximizing the link speed at a given time.For this second task we compare simulation results with the results of experiment executed with the real robot. For each task, we analyze the stiffness modulation and we show that the optimal control approach enhances the explosive motion capabilities by simultaneous modulation of position and stiffness. In order to do that, simulations and experiments are done in two cases. 1) Case-I: When the sum of the pressure in agonistantagonist pair of muscles at each joint is kept constant, i.e. $P_{1}+P_{2}=$ Constant. 1) Case-II: When $P_{1}$ and $P_{2}$ are left independent. In this case, there will be two inputs for each joint. 


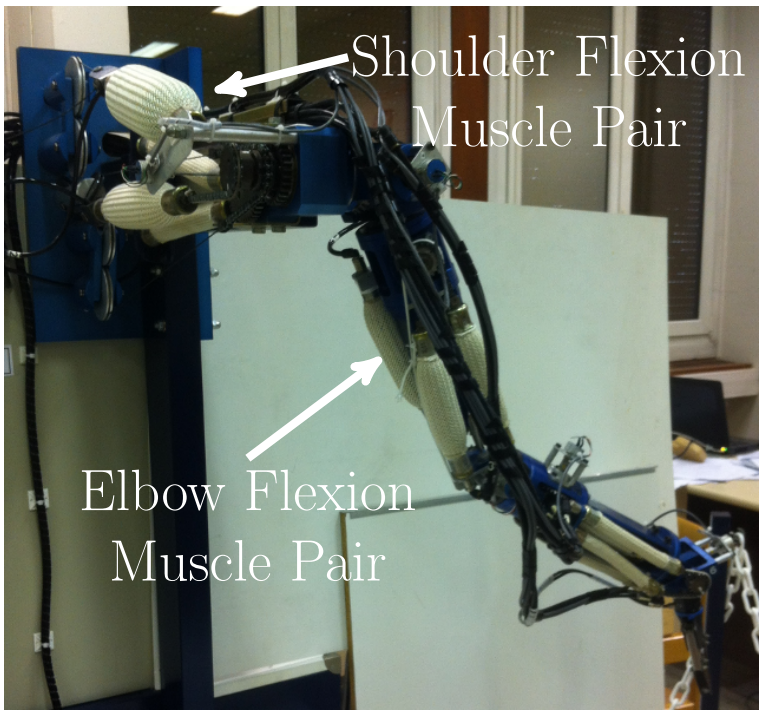

Fig. 5. Picture of the robot-arm showing the two pairs of muscles, in shoulder and elbow flexion.

\section{A. Task 1: Position control}

The manipulator is given a final position with a load mass of $m_{l}=0.1 \mathrm{~kg}$ at the end effector. iLQR is used here to find the optimal path to reach the final goal $\left(\theta_{1}, \theta_{2}\right)=$ $(28.8,57.6)$ degrees. The same task is repeated with different load masses of $m_{l}=0.1 \mathrm{~kg}$ and $m_{l}=1.0 \mathrm{~kg}$ which is shown in Fig. 6.

The following cost function is considered for this task.

$$
\begin{array}{r}
C_{f}=Q_{f}\left(x_{r e f}(T)-x(t)\right)^{2}, \\
C_{r}=Q \int_{0}^{T}\left(\left(x_{r e f}(T)-x(t)\right)^{2}+u^{2}(t)\right) d t,
\end{array}
$$

where, $x_{\text {ref }}$ is the final position for the two joints. The iLQR control uses Eq.(14) and Eq.(19) to solve for optimal control sequence $u^{*}$. This optimal control sequence in forward application to Eq.(14) will yield the needed trajectory. To compare the effectiveness of the iLQR approach, the task is executed using a Proportional-Integral controller with a feedforward term. The feed-forward term gives a desired pressure for each muscle at the joint needed to maintain a desired joint angle. Thus, the control action of the feed-forward PI controller can be defined as follows:

$$
u(t)=P_{\text {feed }}+K_{p}(e(t))+K_{i} \int_{0}^{t}(e(t)) d t
$$

where, $e(t)=x_{r e f}-x(t), K_{p}$ and $K_{i}$ are proportional and integral gains respectively. The simulation results are shown in Fig. 7. Response of feed-forward PI controller is shown in black dashed line is compared with the responses of optimal position control in Case-I when $P_{1}+P_{2}=$ Constant (blue lines) and in Case-II when $P_{1}$ and $P_{2}$ are independent (magenta lines)(See Fig. 7).

From the position plots in Fig. 7, it appears that the iLQR approach gives a good compromise between keeping the stiffness low and minimizing the oscillations which results into a smooth motion. However, the feed-forward PI controller
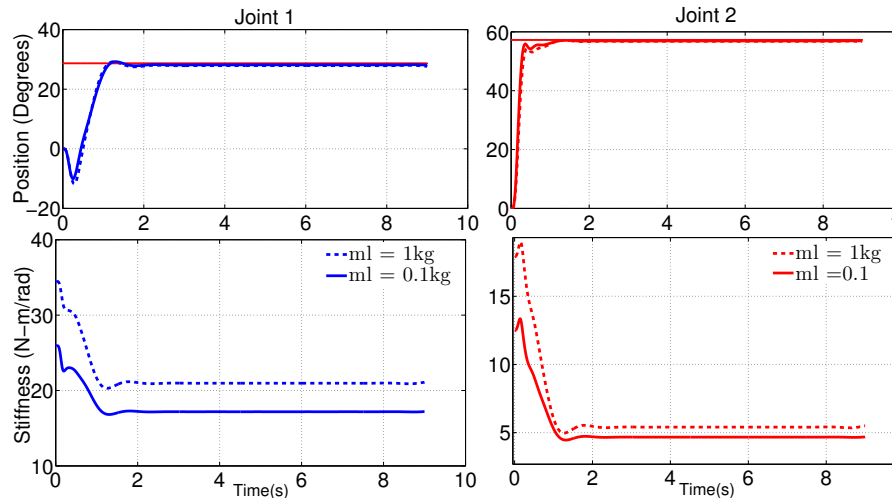

Fig. 6. Figures on the left side represents the position response and stiffness variation at joint 1 and the figures on the right side represents the same for joint 2 in order to reach the target $\left(\theta_{1}, \theta_{2}\right)=(28.8,57.6)$ degrees. The plots compare the response of the joints and the stiffness profile when the end effector is loaded with mass $0.1 \mathrm{~kg}$ (solid line) and $1.0 \mathrm{~kg}$ (dashed line). For the heavier load, optimal control gives a stiffer stiffness profile.
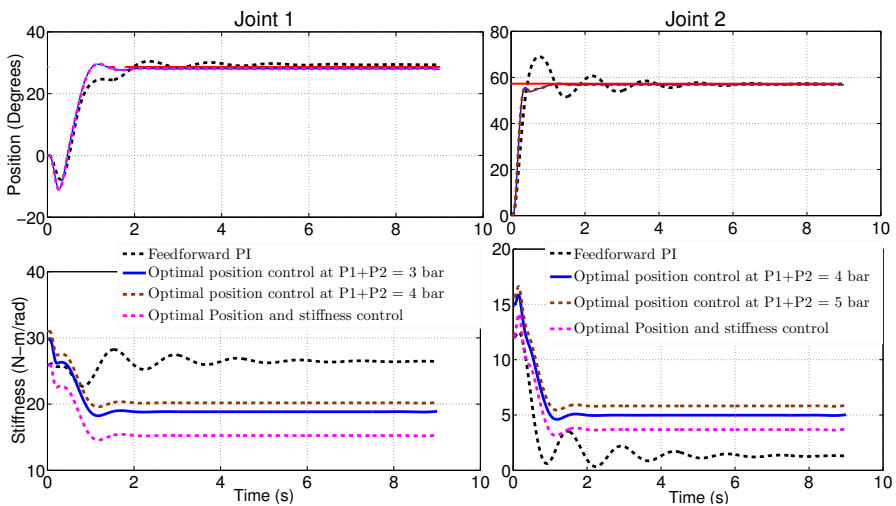

Fig. 7. Figures on the left side represents the position response and stiffness variation at joint 1 and the figures on the right side represents the same for joint 2.

makes joint 1 very stiff and joint 2 very flexible leading to an overshoot and oscillations.

As discussed in section II-C, the stiffness can be adjusted for the same equilibrium position by changing the sum of pressures in the agonist-antagonist pair of muscles. As the sum increases, stiffness at the equilibrium increases which is evident at each joint (blue and brown lines in stiffness plots).

In order to find the most compliant position trajectory, i.e to find the best sum of pressure in the agonist-antagonist pair, $P_{1}, P_{2}$ are left independent and Eq.(7) has two independent inputs for each joint. In this case, the optimal solution gives the similar optimal position trajectory but with better stiffness profile (magenta line in stiffness plot in Fig. 7).

\section{B. Task 2: Maximizing the link speed}

The objective is to execute some explosive motions with the aim to perform in the future tasks such as ball throwing, kicking or hammering a nail. Such motions would require either maximizing the joint/link speed or end-effector speed [17], [18]. Here, two subtasks are presented: 1) Maximizing the angular speed of the elbow joint. The task is first simulated and then executed by the real robot. A comparison between 
simulation and the experimental results are shown in Fig. 8. 2) Maximizing the end effector speed: The objective of this task is to achieve maximum end-effector speed at terminal time $T$. Simulations are done for both Case-I $\left(P 1_{1}+P_{2}=\right.$ Constant $)$ and Case-II ( $P_{1}$ and $P_{2}$ are independent).

1) Maximizing the angular speed: For maximizing the elbow joint's angular speed at final time $T=1$, the task requires only the terminal cost, $C_{f}$. But to minimize the control effort, a running cost $C_{r}$ involving only control pressures is used.

$$
\begin{aligned}
& C_{f}=-Q_{f}\left(x_{4}(T)\right)^{2}, \\
& C_{r}=Q_{u} \int_{0}^{T} u^{2}(t) d t
\end{aligned}
$$

where, $x_{4}=\dot{\theta}_{2}$ is the angular velocity of the elbow joint which constitutes the fourth state variable. $Q_{f}$ and $Q_{u}$ are weights for the terminal cost and the running cost. The simulation results are shown in Fig. 8

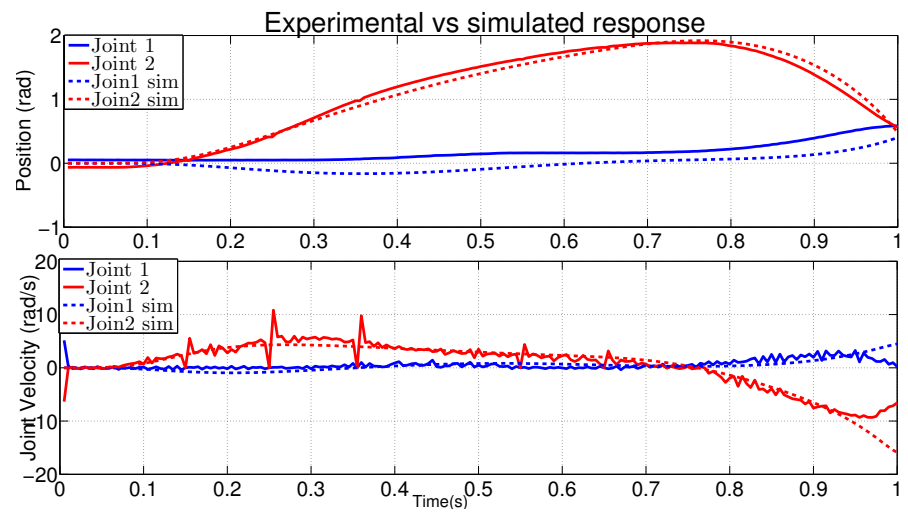

Fig. 8. Plots on the top compare the robot response (solid lines) with simulated response (dashed lines) at joint 1 (blue line) and joint 2 (red line). Bottom plots compare the velocity response of the robot and the simulation.

In simulation, the maximum joint velocity is reached at terminal time which is around $-15 \mathrm{rad} / \mathrm{sec}$. The robot response and the simulation response in position and speed show a good match. However, some discrepancies can be observed. Apart from some modeling error, this difference is probably due to the fact that the constraints on the state vector are not considered in the optimal control problem formulation. This is an improvement that we plan to do in future work. Snapshots of the video of the experiment are presented in Fig. 12

2) Maximizing the end effector speed: The objective is to maximize the end-effector speed of the manipulator at terminal time. The cost function, thus, comprises the terminal cost $C_{f}$ involving the end-effector velocity and the running cost $C_{r}$ involving only control efforts.

$$
\begin{gathered}
C_{f}=-Q_{f}\left(f_{\text {vel }}(x(T))^{2},\right. \\
C_{r}=Q_{u} \int_{0}^{T} u^{2}(t) d t,
\end{gathered}
$$

where, $f_{\text {vel }}(x(t))$ is the function which computes end effector velocity from the robot kinematics. Simulations are done for the following two cases. In Case-I, the sum of pressures in the agonist-antagonistic pair is kept constant at the joint 1 to 3 bar and at the joint 2 to 4 bar. In this second simulation (Case-II),
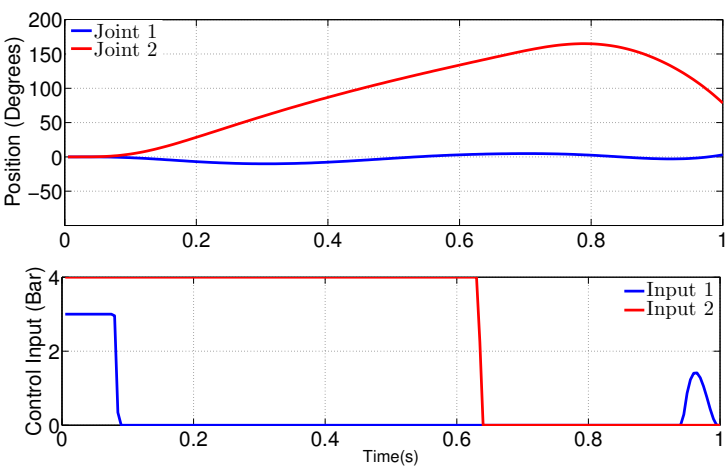

Fig. 9. Case-I: $P_{1}+P_{2}=$ Const. Plots on the top show the simulated position response of joint 1 (blue line) and Joint 2 (black line). Control effort is shown in the bottom plots. The control inputs, Input 1 and Input 2 are applied on joint 1 and joint 2 respectively.
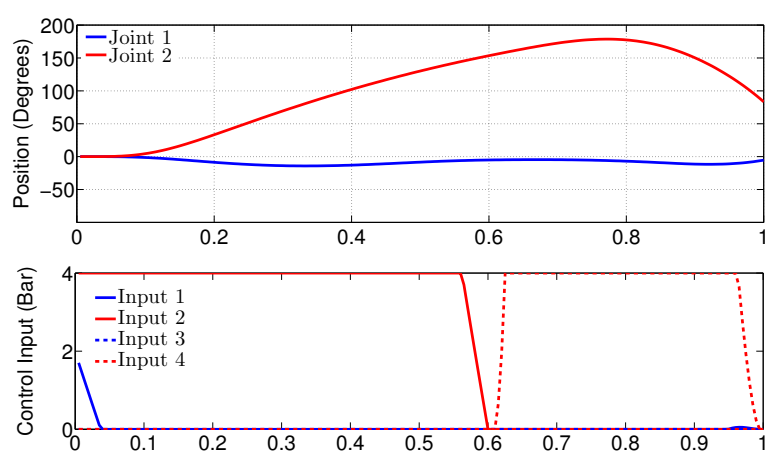

Fig. 10. Case-II: $P_{1}, P_{2}$ are independent. Plots on the top show the simulated position response of joint 1 (blue line) and joint 2 (black line). Control effort is shown in the bottom plots. The control inputs, Input 1 and Input 3 are inputs to the agonist-antagonist pair at joint 1 . The control inputs, Input 2 and Input 4 are inputs to the agonist-antagonist pair at joint 2 .

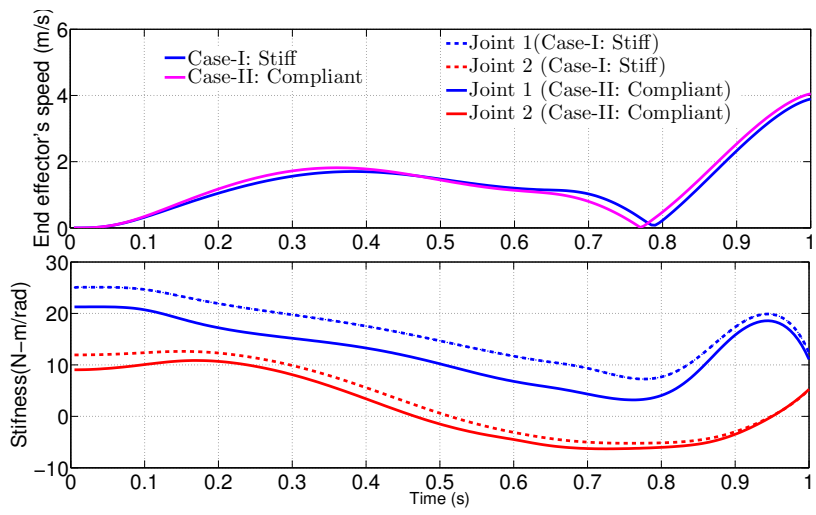

Fig. 11. Comparison of the stiffness profile of each joint (joint 1: blue lines, Joint 2: red lines) in Case-I when $P_{1}+P_{2}=$ Const (dashed lines ) and Case-II when $P_{1}, P_{2}$ are independent (solid lines). It is evident from the plots that the motion in Case-II is indeed slightly more compliant than the motion in Case-I.

we let $P_{1}, P_{2}$ independent.

Comparing the velocity plots and the corresponding stiffness plots in Fig. 11, it can be seen that the maximum end-effector velocity has increased from $3.8 \mathrm{~m} / \mathrm{s}$ in Case-I to 

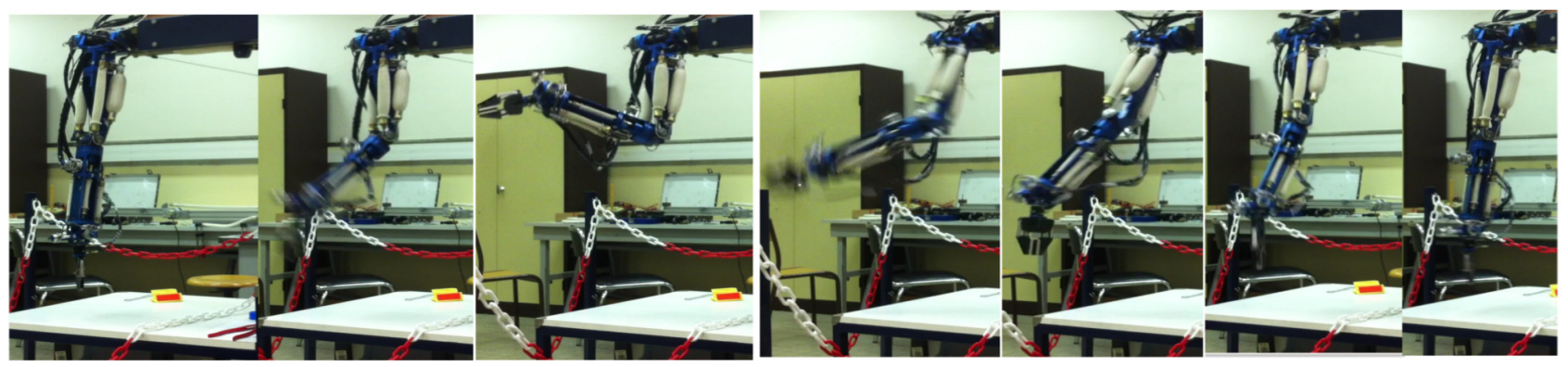

Fig. 12. The pneumatic manipulator arm executing the task of maximizing the angular speed of the elbow.

$4.1 \mathrm{~m} / \mathrm{s}$ in Case-II. This is due to the fact that in Case-II, the optimal control has the freedom to modulate the compliance with two inputs at each joint and hence it was able to achieve higher speed.

The hardware set up used for the experimentation is the robot mentioned in [2] whose control modules have been upgraded recently. The important components of the current set up are I/P converters, encoders, NI data acquisition devices and the development computer running real time control software. 1) I/P converter : It is a Samson I/P 6111 manufactured by Samson Corporation, Frankfurt. This I/P is rapid and produces output pressure in range 0 to 5 bar. The bandwidth of the $\mathrm{I} / \mathrm{P}$ is volume dependent. There are 7 joints and a gripper. Each joint and the gripper are actuated by a pair of Mckibben muscles and each muscle is controlled by one I/P so, there are 16 IP converters. 2) Encoders/Potentiometers: There are seven potentiometers, one at each Dof to get the joint angle data. 3) The data Acquisition device: CompactRIO from National Instruments is used for this purpose. This is a reconfigurable and embeddable chassis, integrating an intelligent real-time controller. It has NI9205 module which is an Analog-to-Digital-Converter (ADC) with up to 32 channels, selectable acquisition range $(200 \mathrm{mV}, 1 \mathrm{~V}, 5 \mathrm{~V}$ and $\mathrm{OV})$ and a max sampling frequency of $250 \mathrm{kS} / \mathrm{s}$. This device samples the seven potentiometers dedicated to the positioning of the arm. There is a NI9265 card which has three modules of Digitalto-Analog-Converters (DAC). They have the particularity to provide analog current outputs. Each module is able to manage up to 4 channels in the 0 to $20 \mathrm{~mA}$ range at $100 \mathrm{kS} / \mathrm{s}$. 4) The development computer: The development computer runs the high level control algorithm for the robot. It communicates via the NI module using standard UDP protocol at a frequency of $1 \mathrm{kHz}$ in real time. The computer has linux 14.04 with Xenomai real time kernel running in parallel. For the present experimentation, control software are running at $200 \mathrm{~Hz}$ frequency.

\section{CONCLUSION AND FUTURE WORK}

We considered the problem of modeling and controlling a robot manipulator actuated by the pneumatic Mckibben muscles in an optimal control frame-work. Our first contribution is to propose a model which encapsulates the pressure dynamics in an efficient way. It is done by using a second order differential function whose bandwidth is dependent on the instantaneous volume of the muscles. Even though, the model does not include the effect of dry friction and hysteresis, it covers most of the static and the dynamic behavior. Using this model, along with the robot manipulator model, our second contribution is to show that the implementation of iLQR allows to perform efficient position control but also preserves the inherent compliance of the Mckibben muscles with respect to the more conventional control approaches. The optimal control formulation with this new model is used to perform various tasks like positioning control under different loads and maximizing the link speed. The results were reported in simulation first and then some of these results were validated on the hardware platform. In future work, we plan to extend the application on the real robot in order to perform explosive movements such as throwing a ball or hammering a nail. This will be done by embedding the state constraints in the optimal control formulation. A detailed analysis of stiffness variation and possibilities of stiffness control will then be possible.

\section{ACKNOWLEDGEMENTS}

This work was partially supported by the European FP7 Project EUROC under Grant Agreement 608849 and the ERC Advanced Grant Actanthrope. We thank Jerome Manhes for his support in hardware development, Florent Forget and Kevin Giraud Esclasse for their help in software development.

\section{REFERENCES}

[1] V. Kumar, Z. Xu, and E. Todorov, "Fast, strong and compliant pneumatic actuation for dexterous tendon-driven hands," in ICRA, 2013.

[2] B.Tondu, S. Ippolito, and J. Guiochet, "A seven-degrees-of-freedom robot-arm driven by pneumatic artificial muscles for humanoid robots," Int. Jour. of Robotics Research, vol. 24, no. 4, 2005.

[3] B. Tondu, K. Braikia, M. Chettoulr, and S. Ippolito, "Second order sliding mode control for an anthropomorphic robot-arm driven with pneumatic artificial muscles," in Int. Conf. on Humanoid Robotics (ICHR), 2009, pp. 47-54.

[4] Y. Tassa, T. Wu, J. Movellan, and E. Todorov, "Modeling and identification of pneumatic actuators," in Int. Conf. on Mechatronics and Automation (ICMA), 2013, pp. 437-443.

[5] V. Kumar, Y. Tassa, T. Erez, and E. Todorov, "Real-time behaviour synthesis for dynamic hand-manipulation," in ICRA, 2014.

[6] G. Andrikopoulos, G.Nikolakopoulos, and S. Manesisa, "Pneumatic artificial muscles: A switching model predictive control approach," Control engineering practice, vol. 21, pp. 1653-1664, 2013.

[7] B. Tondu, "Modelling of the mckibben artificial muscle: A review," Journal of Intelligent Material Systems and Structures, vol. 23, no. 3, 2012.

[8] C. Kothera, M. Jangid, J. Sirohi, and N. Wereley, "Experimental characterization and static modeling of mckibben actuators," Transactions of the ASME, Journal of Mechanical Design, vol. 131, 2009.

[9] B. Tondu and S. Zagal, "Mckibben artificial muscle can be in accordance with hill skeletal muscle model," in IEEE/EMBS Int.Conf, BioMed. Rob. I\& BioMecatron., no. 276, 2006. 
[10] F. Daerden and D. Lefeber, "The concept and design of pleated pneumatic artificial muscles," International Journal of Fluid Power, vol. 2, no. 3, pp. 41-50, 2001.

[11] E. Kelasidi, G. Andrikopoulos, G. Nikolakopoulos, and S. Manesis, "A survey on pneumatic muscle actuators modeling," Journal of Energy and Power Engineering, vol. 6, pp. 1442-1452, 2011.

[12] B. Vanderborght, B.Verrelst, B.Ham, R. V. Damme, M. V. Beyl, and P. Lefeber, "Torque and compliance control of the pneumatic artificial muscles in the biped "lucy"," in ICRA, 2006, pp. 842-847.

[13] V. Boitier, "Design and control of a 2-dof scara-type robot actuated by pneumatic mckibben artificial muscles," Ph.D thesis, INSA-Toulouse, France, 1996.

[14] B. Tondu and P. Lopez, "Theory of an artificial pneumatic muscle and application to the modelling of mckibben artificial muscle," C.R.A.S. French National Academy of Sciences, vol. 320, no. 4, 1995.

[15] Y. Tassa, N. Mansard, and E. Todorov, "Control-limited differential dynamic programming," in ICRA, 2014.

[16] W. Li and E. Todorov, "Iterative linearisation methods for approximately optimal control and estimation of non-linear stochastic system," Int Jour. of Control, vol. 80, no. 9, pp. 1439-1453, 2007.

[17] M. Garabini, A. Passagliaand, F. Belo, P. Salaris, and A.Bicchi, "Optimality principles in variable stiffness control: Vsa hammer," in IROS, 2011.

[18] D.Braun, F. Petit, F. Huber, S. Haddadin, P. D. Smagt, A. Albu-Schaffer, and S.Vijayakumar, "Robots driven by compliant actuators: Optimal control under actuation constraints," in IEEE Transactions on Robotics, vol. 29, 2013. 